\title{
Educação Física, Qualidade de Vida e Saúde: campos de intersecção e reflexões sobre a intervenção
}

Prof. Ms. Fabiano Pries Devide*

\section{Resumo}

Este ensaio tem por objetivo refletir sobre as relações entre a Educação Física a Qualidade de Vida e a Saúde. Posiciona a Saúde como foco da discussão, considerando-a área de intersecção entre as duas primeiras, elemento-chave nas representações sociais sobre a Educação Física, e possivelmente sobre a Qualidade de Vida. São discutidos aspectos relacionados à complexidade $\mathrm{e}$ dinamicidade do que se denomina Qualidade de Vida; sobre as relações entre a Educação Física e a Saúde na escola; assim como suas contribuições para uma reflexão acerca do neologismo da Qualidade de Vida e as relações recentemente estabelecidas com a área. Propõe-se uma reorientação sobre o entendimento destas relações, em direção à superação da noção de causalidade entre Exercício-Saúde-Qualidade de Vida, através da fragilização das responsabilidades $\mathrm{e}$ compromissos da Educação Física frente às questões relacionadas à melhoria da Qualidade de Vida e da Saúde.

Descritores: Educação Física, Qualidade de Vida, Saúde, Escola.

\section{Abstract}

The objective of this paper is to think the relations between Physical Education, Quality of Life, and Health. Health is thought of as the central topic ofthe discussion, considering it as an intersection área between the other two. It is also considered as a key element of social representations of Physical Education, andprobably, of Quality of Life. The paper also points outsome aspects relatedto the complex and dynamic characteristics ofthe concept of Quality of Life; the relations between Physical Education and Health in the School; and its contributions to rethink about this new term Quality ofLife and the relations recently established with the área. The paper also suggests a re-orientation on the understandingofthese relations, overcoming the notion of causality between exercise-health-quality of life, through the idea of fragility ofthe responsibilities of Physical Education and its commitments with the questions relatedto the improvement ofQuality ofLife and Health.

Key Words: Physical Education, Quality of Life, Health, School.

\section{Introdução}

A busca por uma melhor qualidade de vida tem sido uma constante nas sociedades. Isto tem gerado tentativas de se estabelecer valores para o nível de qualidade de vida, a partir de indicadores econômicos, taxas de natalidade, mortalidade infantil, esperança de vida, alfabetização, consumo alimentar, prática de atividade fisica, entre outros aspectos que traduzem sua complexidade, enredada que está com questões de ordem social, como meio-ambiente, educação, segurança e promoção da saúde.

Nesse sentido, as relações circulantes entre atividade física e a qualidade de vida, são empíricas e testemunhadas por nós no quotidiano, como por exemplo, as propagandas de centros de atividade física, que se utilizam desse neologismo para vender os seus produtos.

A qualidade de vida surge como algo a se alcançar. Algo que a prática do exercício físico pode fornecer. Um es- 
tado que, por vezes, emerge relacionado à atividade fisica, através de mensagens veiculadas na forma de imagens ou palavras.

Mas o que podemos chamar de 'qualidade de vida'? Conceituar esta expressão tão escorregadia seria, certamente, trabalho para os filólogos. Como pesquisador que vem investigando as representações sociais sobre objetos que circulam em nosso quotidiano, e influenciam nossas práticas, resta a curiosidade de saber como as pessoas representam a qualidade de vida.

A qualidade de vida emerge sob uma subjetividade que torna difícil a conceituação estrita do termo, que se refere, sobretudo, à história pessoal de cada um de nós; quero dizer, ao que valorizamos como aquilo que é bom para que vivamos nosso dia a dia de forma positiva.

Por isso, se torna relevante o estudo das representações sociais sobre a qualidade de vida daqueles com os quais iremos intervir. E preciso identificar qual o grau de associação que as pessoas estabelecem entre a Educação Física ou a atividade física e o que se tem chamado qualidade de vida. Considerando que essas representações contribuem para alterar os comportamentos dos indivíduos, se desejamos que nossa intervenção seja bem sucedida, nós, professores/as, devemos oferecer aquilo que certamente melhorará a qualidade de viver dos nossos destinatários.

Isso pressupõe alterar o estilo de vida de pessoas, seja através de uma atividade física orientada, no nosso caso; ou de uma leitura, uma ida ao cinema, um bom cochilo após o almoço, um fim de semana na praia; enfim, um infinito de opções em que a Educação Física surge, no mínimo, como mais uma contribuição, ao lado de outras áreas de intervenção como o Turismo, a Sociologia, a Gerontologia, a Psicologia, as Artes, entre outras.

A qualidade de vida tem dinâmica própria, por estar relacionada à cultura local, fazendo com que se deva respeitar as singularidades de cada comunidade e indivíduo em si, evitando padrões conceituais. E preciso discutir o 'ter' qualidade de vida entre aqueles com os quais trabalhamos, para que possamos iden- tificar a dinamicidade desse conceito, que possivelmente traz elementos compartilhados num grupo, mas também singulares a cada uma das pessoas que nele se insere.

\section{Saúde e Educação Física escolar: Tendências Teóricas em construção}

Com o objetivo de discutir este tema no âmbito da Licenciatura em Educação Física, organizei algumas idéias acerca da Educação Física escolar, da qualidade de vida e da saúde; esta última, posicionada como aspecto central, em virtude de considera-la uma área de intersecção entre as duas primeiras, e presente como um elemento chave nas representações sociais sobre a Educação Física, e possivelmente a qualidade de vida.

Estudando o conceito e a construção teórica sobre a saúde e suas relações com a Educação Física, especificamente no campo escolar, identifiquei duas tendências, que considero centrais, e que nas últimas décadas representam duas formas específicas de se interpretar como a Educação Física, na escola, pode contribuir para que alunos possam beneficiar sua saúde (Devide, 2000).

Diante da produção acadêmica existente, um ponto se mostra consensual: a Educação Física e a saúde possuem uma relação histórica (Faria Júnior, 1991a; Soares, 1994; Carvalho, 1993;Anjos, 1997); mas o grau de relacionamento se modifica conforme nos deparamos com as discussões propostas pelos intelectuais, que de um lado buscam fortalecê-la e de outro atenuá-la.

A 'Promoção da Saúde' (Health Promotion), amplia a relação da Educação Física com a saúde, interpretando a última de forma crítica, reconhecendo seus diversos fatores influenciadores, além da prática de exercícios fisicos (WHO, 1984; Fariajúnior 1991a, 1991b). Outra tendência, a Aptidão Física Relacionada à Saúde (Health Related Fitness), estabelece uma relação entre a Educação Física e a saúde pela via da aptidão, priorizando parâmetros fisiológicos (Corbin e Fox, 1986; Biddle, 1987;Nahas, 1989; Nahas e Corbin, 1992;Corbine Pangrazzi, 1993; Guedes e Guedes, 1993,1994). 


\subsection{A Promoção da Saúde}

E a partir de alguns pressupostos provenientes do ideário da Promoção da Saúde, que gostaria de refletir sobre como nós, professores/as de Educação Física, devemos interpretar com cautela, o discurso e as ações que assumimos quando utilizamos o termo qualidade de vida' como um efeito da prática de atividade física orientada por nós. Dentre os diversos textos brasileiros que se aproximam desta abordagem, considero o de Fariajúnior (1991 a) o mais denso. A discussão feita por este autor, se deu a partir de um documento produzi-do pela Organização Mundial daSaúde(WHO, 1984²).

A Promoção da Saúde, aborda quatro questões básicas sobre a saúde: a sua multifatoriedade ${ }^{3}$, a desmedicalização ${ }^{4}$, a educação para a saúde e o seu caráter coletivo $^{5}$, o que pode ser observado em seus cinco princípios, a saber: A Promoção da Saúde (1) deve focalizar a população como um todo e não apenas os grupos de risco; (2) a ação deve ser voltada para muitos fatores que influenciam a saúde; (3) deve envolver uma variedade de estratégias e agências - comunicação, educação, legislação, desenvolvimento comunitário, entre outras; (4) requer a participação de toda a comunidade, envolvendo a aquisição - individual e coletiva de estilos de vida; (5) requer que profissionais da saúde tornem viável a Promoção da Saúde pela sua defesa educação (WHO, 1984).

Esta proposta, já é conhecida e tem sido abordada por alguns pesquisadores na área de Educação Física (Fariajúnior, 1991a, 1991b; Ferreira, 1993,2001; Devide, 1996a, 1996b; Devide, Ferreira, 1997; Carvalho, 2001), que interpretam este conjunto de idéias como rico suporte para discussão do binômio exercício-saúde. Mas acredito que seus pressupostos também podem enriquecer a discussão entre a Educação Física e a Qualidade de Vida.

Dentre os quatro pontos básicos, quero sublinhar a 'educação para a saúde (Mota, 1992), que, primeiramente, contribui para desmedicalizar a saúde, reconhecendo que, além do médico, o trabalho conjunto dos diversos profissionais deve ter como objetivo 'educar' as pessoas a adotar práticas que otimizem sua saúde e a de sua comunidade. A saúde torna-se uma questão didáticopedagógica. Isso significa que os conhecimentos transmitidos, formalmente na escola ou informalmente na comunidade, tendem a fazer com que pessoas assumam atitudes que levem a uma melhor qualidade de vida, desenvolvendo sua auto-capacitação (Tones, 1986) ${ }^{6}$.

A Educação Física tem a possibilidade de ampliar o alcance de seus conteúdos, se em alguns casos, buscar discutir aspectos relacionados à educação para a saúde, através da aquisição, por alunos, de estilos de vida ativos e hábitos saudáveis. Esse estilo de vida extrapola a idéia de adesão à prática regular de exercícios físicos; deve se referir à aquisição de hábitos que otimizem o seu status de saúde e de sua comunidade, pois "a participação da comunidade amplia a concepção de estilos de vida do nível individual para o coletivo, e exige da educação física novas responsabilidades, papel e ações interativas" (Faria Júnior, 1991 a, p. 26).

Uma Educação Física compromissada com a melhoria da Qualidade de Vida deve levar os alunos a se exercitarem, a desenvolverem conhecimentos sobre a prática física, e sobretudo a se conscientizarem da sua importânciaebenefíciosparaavida, possibilitando identificarem os fatores que os impedem, por vezes, de praticar exercícios regularmente e melhorarem sua qualidade de vida.

\subsection{A Aptidão Física Relacionada a Saúde e a propos- ta da Escada da Aptidão para Toda a Vida}

Outros intelectuais abordam a relação da Educação Física com a saúde, basicamente através da aptidão físi$\mathrm{ca}^{7}$, consubstanciados pelos benefícios fisiológicos dos exercícios, provocados pela adoção de um estilo de vida ativo. Discorrerei basicamente sobre a proposta de Charles Corbin ${ }^{8}$, corroborada por autores nacionais, como Markus Nahas (1989) e Dartagnan Guedes (1993, 1994).

Os estudos dos autores supracitados abordam a questão da Educação Física com a saúde no espaço escolar sob as bases da Aptidão Física Relacionada à Saúde (Health Related Fitness) (Biddle, 1987). Esta cor- 


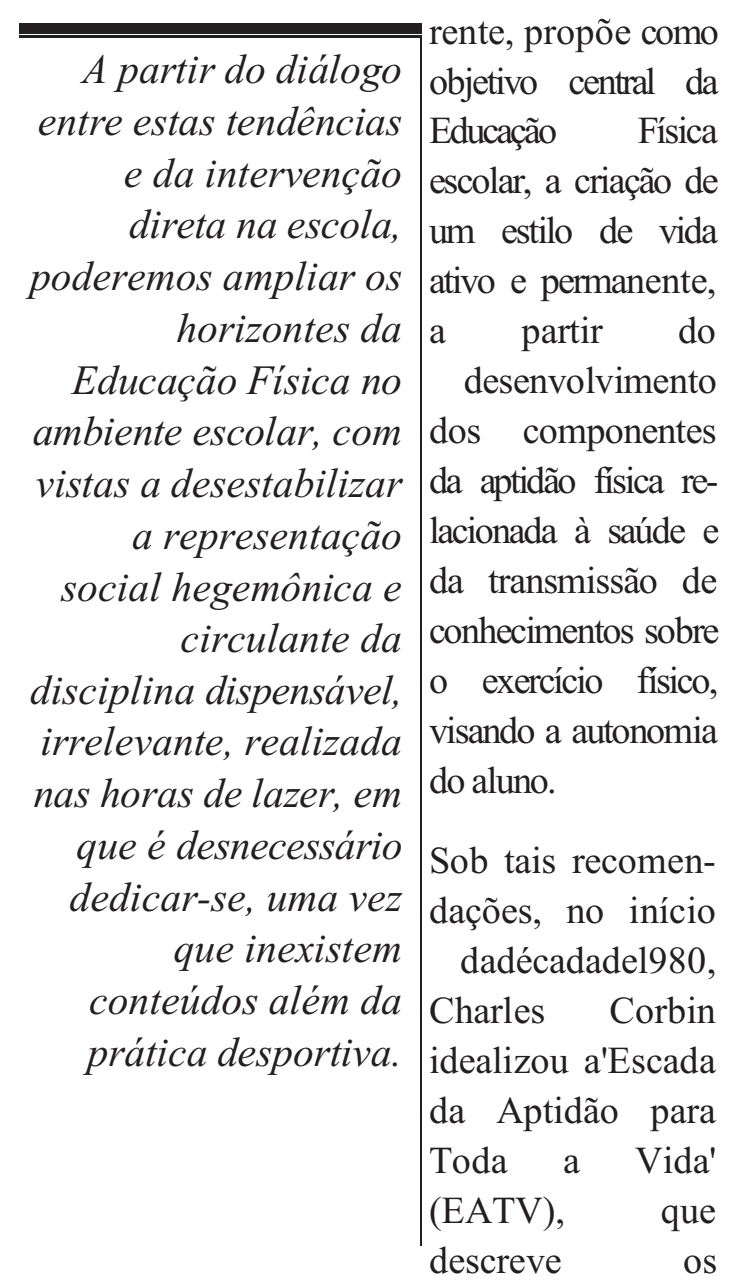

objetivos da Educação Física no ambiente escolar. A proposta se desenvolve em fases, simbolizadas por degraus, a serem transpostos no decorrer dos anos.

O primeiro degrau é representado pelas atividades fisicas e os exercícios. Tem como objetivo tornar os alunos ativos nos primeiros anos escolares, despertando o prazer pelo exercício. O segundo diz respeito à aquisição de aptidão fisica, que só será desenvolvida caso o aluno seja fisicamente ativo.

Para se desenvolver estilos de vida ativos e permanentes, outros objetivos são incluídos na proposta. E demarcada uma linha pontilhada entre o segundo e terceiro degraus, para sublinhar a atitude de professores que se satisfazem quando os seus alunos atingem níveis de aptidão satisfatórios. A questão que se formula é: os alunos, apesar de aptos fisicamente, adotarão um estilo de vida ativo, uma vez que não conhecem os benefícios do exercício, e como progra- mar e avaliar programas?

Por isto, há progressão em relação aos compromissos da Educação Física e, no terceiro degrau, conhecimentos referentes à aptidão física e ao exercício devem ser transmitidos ao aluno, que inicia a construção do seu próprio esquema de exercícios, desenvolvendo certa 'autonomia' em relação à figura do professor. $\mathrm{O}$ quarto degrau refere-se à auto-avaliação da aptidão física. Os alunos são sujeitos a testes, estabelecendo objetivos individuais, para avaliarem os componentes da aptidão relacionada à saúde, interpretando os seus resultados no planejamento de seu programa de exercícios.

Quando se atinge o último degrau da EATV, pressupõese: o aluno é preparado para solucionar problemas $e$ tomar decisões a respeito de sua prática de exercícios; já experimentou diversas atividades; pode escolher a(s) que aprecia e incorpora-la(s) em seu quotidiano, tendo conhecimento suficiente para tornar-se um consumidor bem informado a respeito do exercício físico.

O ponto mais importante enfatizado por esta proposta, é a autonomia criada pelo aluno sobre o planejamento de seu programa de exercício, exercitando-se ciente dos seus benefícios para sua saúde. Assim, não basta desenvolver bons níveis de aptidão física relacionados com a saúde; propõe-se trabalhar aspectos cognitivos referentes à aptidão e aos exercícios, para que os alunos tomem atitudes a respeito da criação de estilos de vida ativos e permanentes, com prazer e conscientes de seus benefícios.

\section{Reflexões sobre estas tendências:}

Ambas as tendências acima buscam desenvolver um estilo de vida ativo; e por diferentes enfoques, nos fazem refletir sobre reorientações que a Educação Física Escolar pode assumir na sua relação com a saúde e enquanto veículo de educação para a saúde (Devide, 1996b).

Ambas trazem contribuições, mas também limitações. A Promoção da Saúde, representada no Brasil, basica- 
mente pela produção teórica de Faria Júnior (1991a, 1991 b), clarifica a relação tênue entre a Educação Física e a saúde; no entanto, não apresenta uma intervenção prática de como se tratar e aplicar os seus pressupostos na escola; o que começa a surgir lentamente com o esforço de outros autores (Oliveira, Devide, 2000; Ferreira, 2001) e pode ser observado, mesmo que de forma sutil, em práticas pedagógicas de alguns profissionais que atuam no ambiente escolar.

Quanto à Aptidão Física Relacionada à Saúde, alguns estudos têm se esforçado para sistematizar a proposta. Dentre a produção encontrada no Brasil, dois artigos de Dartagnan Guedes e Joana Guedes $(1993,1994)$ foram os que melhor sistematizaram, com clareza, a proposta da EATV. Os autores buscam dar suporte e sugestões de conteúdo para a implementação de programas de Educação Física escolar direcionados à promoção da saúde, focados na segunda tendência apresentada.

As críticas feitas à proposta de criação de um estilo de vida ativo pela Aptidão Física Relacionada à Saúde, são: o reducionismo da saúde ao seu aspecto biológico; a individualização do problema centrado no aluno, que se torna responsável pelo desenvolvimento de sua aptidão e melhoria de sua saúde, contribuindo para o processo de culpabilização da vítima ${ }^{10}$; o etapismo, inerente aos conteúdos, dificultando o entendimento sobre o binômio exercício-saúde de forma ampliada, considerando-se outros aspectos além do exercício; e a repro-. dução da relação causai que se construiu na história, sobre a prática de exercício e a conseqüente melhoria da saúde (Devide, 1996a; Ferreira, 2001).

E importante se propor uma abordagem, que estabeleça a tensão entre estas tendências, relacionando a saúde, concebida de forma multifatorial; com a Educação Física, enquanto veículo de educação para a saúde. A partir do diálogo entre estas tendências e da intervenção direta na escola, poderemos ampliar os horizontes da Educação Física no ambiente escolar, com vistas a desestabilizar a representação social hegemônica e circulante da disciplina dispensável, irrelevante, realizada nas horas de lazer, em que é desnecessário dedicar-se, uma vez que inexistem conteúdos além da prática desportiva. O início da construção de uma Educação
Física que se legitime no espaço escolar, pode se dar com o desenvolvimento de conteúdos relevantes para o quotidiano dos alunos, construídos coletivamente na interação da sala de aula (Betti, 1995;Devide, 1999; Devide; Rizzuti, 1999).

Na busca de transformações nas representações sociais dos alunos sobre a Educação Física, uma preocupação central deve ser a ampliação da questão da sua relação com a saúde, a partir da produção científica já existente e da interação entre profissionais atuantes e comprometidos, o que favorece a Educação Física como veículo potencial de educação para a saúde, visando a criação de estilos de vida ativos e permanentes, contribuindo para uma melhor qualidade de vida de alunos, consumidores críticos dos elementos da cultura corporal.

\section{Pressupostos que contribuem para a discussão no âmbito da qualidade de vida}

Apesar da dificuldade de consenso sobre o que seja qualidade de vida, o termo relaciona-se ao estilo de vida de pessoas que priorizam e buscam suprir diferentes necessidades que contribuem para a conquista ou manutenção de uma forma melhor de se viver. Nesse contexto, a Educação Física é considerada um meio para o seu aprimoramento; o que pode ser feito através de mudanças nesse estilo de vida, tornando-os mais saudáveis, ativos e permanentes.

O objetivo presente nas tendências apresentadas, de se desenvolver estilos de vida ativos e permanentes; além da discussão sobre o traço multifatorial do conceito de saúde; a estratégia de educação para a saúde; e a dinamicidade do entendimento do que possa ser considerada qualidade de vida; traz novos elementos para aqueles profissionais que atuam com a Educação Física escolar.

O professor deve contextualizar os conteúdos da disciplina à realidade de seus alunos, no sentido de tornálos críticos frente às suas condições de vida. Ao discutir com eles os diversos fatores, além dos exercícios físicos, que influenciam na sua qualidade de vida (a falta 
Ao discutir com eles os diversos fatores, além dos exercícios físicos, que influenciam na sua qualidade de vida (a falta de espaços públicos para a prática de exercícios,

a dificuldade de acesso ao lazer, as más condições de trabalho, transporte, educação etc), contribuirá para a desmitificação da relação equivocada que vem se reproduzindo entre este neologismo e o exercício fisico, ampliando a relação de compromisso da Educação Física como veículo de educação para a saúde. condições políticas, sociais e econômicas (PEREIRA, 1988; Palma, 2001). Como disciplina escolar, aEducação Física "(...) não deve abandonar sua preocupação em subsidiar e encorajar as pessoas a adotarem estilos de vida ativa. Porém, esse seu papel estará limitado se ela não for capaz de promover o exame crítico dos determinantes sociais, econômicos, políticos e ambientais diretamente relacionados aos seus conteúdos". (Ferreira,2001,p. 49-50).

A prática de exercícios físicos, portanto, não pode ser encarada como a solução para todos os problemas de saúde ou para a garantia de uma melhor qualidade de vida das pessoas.
Faz-se necessário ampliar as representações sociais que professores de Educação Física têm a respeito da Educação Física, da saúde e da sua relação com a profissão, pois fundamentam seu trabalho profissional e têm contribuído para perpetuar a noção de causalidade entre o exercício e a saúde, e também entre um estilo de vida ativo e uma boa qualidade de vida. Em estudo com formandos em Educação Física, os dados obtidos permitiram afirmar que uma grande parcela dos futuros profissionais admite uma relação causai entre o exercício e a saúde; desconsideram ou não aprofundam a relação dos fatores sociais, políticos e econômicos que influenciam no 'status' de saúde; e limitam-se a relacionar a Educação Física com a saúde pela via da aptidão física (Devide, Ferreira, 1997).

Enquanto educador, o profissional da área deve estar ciente das implicações de uma concepção equivocada frente ao relacionamento da Educação Física com a saúde e a qualidade de vida. O professor de Educação Física deve estar atualizado ao conceito multifatorial da saúde e à dinamicidade do que pode ser interpretado por qualidade de vida, para que, munido de instrumentação teórica consistente, tenha condições de discutir e ampliar a relação de compromisso da Educação Física para além da esfera da aptidão física.

O professor de Educação Física deve estar atualizado ao

Reconhecer, pri- conceito multifatorial meiramente, que da saúde è̀ nosso papel é parcial dinamicidade do que neste processo, que pode ser interpretado está relacionado aos por qualidade de vida, múltiplos aspectos para que, munido de da vida em instrumentação teórica sociedade, toma-se o consistente, tenha ponto de partida para uma intervenção bem sucedida. A partir dessa reorientação paradigmática, em que a Educação Fí- condições de discutir e ampliar a relação de compromisso da Educação Física para além da esfera da aptidão física. 
sica assume a tarefa de desenvolver estilos de vida ativos, permanentes e conscientes, a Educação Física Escolar poderá legitimar o seu papel em relação à saúde e à promoção da qualidade de vida de forma satisfatória.

\section{Referências}

BETO, I. C. R Educação Física Escolar a Percepção Discente. Revista Brasileira de Ciências do Esporte. Santa Maria. V. 16,n.3,p. 158-167,1995.

BIDLLE, S. (ed.). Foundations of health-related fitness in physical education. London: Ling Publishing House, 1987.

CARVALHO, Y. M. O "mito"da atividade fisica e saúde. São Paulo: Hucitec, 1997.

. Atividade Física e saúde: onde está e quem é o "sujeito " da relação? Revista Brasileira de Ciências do Esporte. Campinas: Autores Associados. V. 22, n. 2, p. 9-21, 2001.

CORBIN, C, PANGRAZZL R Physical fitness: questions teachers ask. JOPERD. Virgínia. V. 64, n. 7, p. 14-19,1993.

CORBIN, C; FOX, K A aptidão para toda a vida. Revista Horizonte. Lisboa. V. II, n. 12, p. 205-208,1986.

DEVIDE, F. P. A Escada da Aptidão para toda a vida sob o enfoque da Promoção da Saúde: uma análise crítica. Anais do $11^{\circ}$ Congresso Internacional de Educação Física/FIEP. Foz do Iguaçu, p. 57, 1996a.

.Educação fisica e saúde: em busca de uma reorientação para a sua práxis. Revista Movimento. Porto Alegre, ano III, n. 5, p. 44-55,1996b.

. As aulas de Educação Física Escolar sob a ótica de seus atores. Sprint. Rio de Janeiro. XVIII, n. 105, p. 3440,1999.

Tendências teóricas a respeito da relação histórica entre a Educação Física e a saúde. Anais do VII Congresso Brasileiro de História da Educação Física, Esporte, lazer e Dança. Gramado, p. 401-407, 2000.

DEVIDE, F.P.;FERREIRA, M.S.Exercício e saúde: a percepção discente - um estudo exploratório. Revista Brasileira de Atividadloranópolis: UFSC, 1989.
NAHAS.M-.CORBIN.C. Aptidão física e saúde nos programas de educação física: desenvolvimentos recentes e tendências internacionais. Revista Brasileira de Ciência e Movimento. São Caetano do Sul. V. 6, n. 2, p. 47-58, 1992.

OLIVEIRA, G. A S. de; DEVIDE, R P. Implementação de uma proposta temática na educação fisica escolar no ensino fundamental: um relato de experiência. Anais do $I V$ Encontro Fluminense de Educação Fisica Escolar: planejando a Educação Física na escola. UFF. p. 53-56, 2000 .

PALMA, A Educação Física, Corpo e saúde: uma reflexão sobre outros modos de olhar. Revista Brasileira de Ciências do Esporte. Campinas: Autores Associados. V. 22, n. 2, p. 2339,2001 .

PEREIRA, F. M. Dialética da Cultura Física. São Paulo: Ícone, 1988.

SOARES, C. L. Educação fisica: raizes européias no Brasil. Campinas: Autores Associados, 1994.

TONES, K Health education and the ideology of health promotion: a review of altemative approaches. Health Education Research. V. 1, n 1, p. 3-12,1986.

WHO. Health promotion: a discussion documentof the concept and principles. Copenhagen: WHO, 1984.

\section{Notas}

${ }^{1}$ Professor da Secretaria Estadual de Educação do Rio de Janeiro; professor da Secretaria Municipal de Educação de Teresópolis; mestre em Educação Física e Cultura pela UGF/RJ; doutorando em Educação Física e Cultura pela $\mathrm{UGF} / \mathrm{RJ}$.

${ }^{2}$ Intitula-se Health promotion: a discussion document of the concept and principies.

${ }^{3}$ Aspecto que reconhece a saúde como um estado resultante de fatores múltiplos, como renda, habitação, acesso à educação, saneamento básico, lazer, trabalho, transporte, acesso aos serviços de saúde, entre outros aspectos.

${ }^{4}$ Interpreta a saúde como área que envolve diversos campos de conhecimento, reunindo o esforço de diversos profissionais. 
${ }^{5}$ Parte do pressuposto que a transmissão de conhecimentos à comunidade contribui para que tenha responsabilidade sobre sua saúde, explicitando seus problemas, reivindicando e sugerindo soluções

${ }^{6} \mathrm{O}$ O Modelo de Auto-Capacitação na educação para a saúde consiste em transmitir o conhecimento necessário para uma tomada de decisão da comunidade. Incorpora a idéia de que a mudança democrática só pode ocorrer pela capacitação de indivíduos para modificar o seu meio; mas há dúvidas se a aquisição de conhecimentos pode garantir mudanças por parte da comunidade.

${ }^{7} \mathrm{~A}$ aptidão física é classificada a partir de seus componentes. A aptidão relacionada às habilidades compreende qualidades como coordenação, equilíbrio, velocidade, tempo de reação e agilidade, que aprendidas, tornam-se permanentes, pois são altamente determinadas por fatores genéticos. A aptidão relacionada à saúde compreende qualidades como resistência cardiorespiratória e muscular, força, composição corporal e flexibilidade, mantidas com a adoção de um estilo de vida ativo, embora também sofram determinações genéticas em algum grau (Corbin, Fox, Whitehead,1987).

${ }^{8}$ Esta proposta é discutida em alguns artigos deste autor com colaboradores (Corbin e Fox, 1986; Nahas e Corbin, 1992; Corbine Pangrazzi, 1993).

${ }^{9}$ Esta autonomia é relativa e se refere aos conhecimentos básicos a respeito dos benefícios da prática física, da fisiologia, biomecânica, da nutrição, da história, que capacitam os alunos a 'consumir' o exercício e todos os produtos atrelados à prática física de forma crítica, recusando assumir práticas enganosas, e valorizando os profissionais com formação na área.

${ }^{10} \mathrm{O}$ processo de 'culpabilização da vítima' consiste em depositar no cidadão, a responsabilidade por sua saúde individual, ignorando os múltiplos fatores que atuam sobre sua saúde e dos quais ele não possui controle.

Recebido em: 17.10.01

Revisado em: 22.03.02

Aceito em: 10.05.02 